\title{
Leptospirosis asociada a la exposición ocupacional: Estudio clínico y epidemiológico
}

\author{
MARCELO CARNEIRO, M. DE LOURDES GIACOMINI y J. MARGARETE COSTA
}

\author{
Leptospirosis outbreak related to occupational exposure: Clinical and \\ epidemiological study
}

Objective: to study the behavior of a leptospirosis outbreak that occurred among workers of the Veterinary Hospital - Brazil from October 1998 to January 1999. Method: Prospective investigation of a leptospirosis outbreak related to occupational exposure. Results: From a total of 37 patients, 20 (54.1\%) were males. Individuals exerting different activities were infected during the outbreak, the majority of them (24.3\%) being cleaning-workers, followed by those exerting general functions (18.9\%). The Leptospira serotypes found most frequently were bratislava, (n: $25,43.1 \%)$ and L. australis, (n: 24, 41.4\%). Conclusions: Regarding the clinical aspects of the outbreak, we have found the poly-symptomatic form to be the most prevalent, with no report of any case of serious illness. The highest prevalence of cases among the cleaning-workers of the HV-UFSM rises suspicion at the lack of information about the ways of acquisition of this zoonosis.

Key words: Leptospirosis; Occupational exposure; Epidemiology.

Palabras claves: Leptospirosis; Exposición ocupacional; Epodemiología.

\section{Introducción}

La leptospirosis es una enfermedad aguda y generalizada caracterizada por una vasculitis infecciosa que provoca innumerables alteraciones en órganos nobles como pulmones, hígado y riñones ${ }^{1-4}$.

Afecta al hombre y a los animales en forma endémica, representando un problema de salud pública en los países latinoamericanos ${ }^{2,3}$. La enfermedad se presenta en brotes epidémicos en países subdesarrollados o en vías de desarrollo, principalmente en épocas de lluvias e inundaciones, remarcando su carácter estacional y distribución cosmopolita.

El hombre es introducido en la cadena epidemiológica de modo accidental (hospedero transitorio), por contacto con el agua (importante medio de transmisión), suelo, o exposición directa a las excretas contaminadas de algunos roedores o animales domésticos y salvajes, infectados, adquiriendo la infección y enfermando algunas veces. Es rara la transmisión de persona para persona, pues el hombre representa el final de la cadena de transmisión ${ }^{3}$.

La rata o ratón de alcantarilla (Rattus norvergicus) y el ratón negro o ratón del techo (Rattus rattus) son considerados reservorios ecológicos de la leptospirosis, no desarrollando síntomas de esta zoonosis ${ }^{3}$.

En Brasil, la leptospirosis afecta principalmente a adultos jóvenes del sexo masculino (20-30 años) sucediendo en las estaciones calientes y lluviosas. La prevalencia de esta zoonosis está en aumento, fuertemente relacionado con el empeoramiento de las condiciones de vida y trabajo de la mayoría de la población. De este modo no es considerada sólo de carácter ocupacional, sino también del ámbito social.

En el período de 1991 a 2000 fueron confir-

Servicio Médico de Enfermedades Infecciosas

Hospital Universitário de Santa María

Universidad Federal de Santa María, Rio Grande do Sul. Brasil.

Recibido: 13 enero 2004

Aceptado: 5 octubre 2004 
mados 34.142 casos de leptospirosis en Brasil, con una media anual de 3.414, variando entre 1.728 (1993) y 5.579 casos (1996). En este periodo fueron reportadas 3.274 muertes, con una media de 327 muertes/año y cifras extremas de 215 (1993) y 439 (1998). La tasa de letalidad en este periodo fue de $10,2 \%$, oscilando entre $6,6 \%$ (1996) y $13,8 \%$ (1992). La incidencia fue de 2,2/100.000 habitantes, variando de 1,14 (1993) a 3,55 (1996) ${ }^{3}$. El Estado de Rio Grande do Sul alcanza una alta incidencia de leptospirosis, cercana a 10 casos por 100.000 habitantes, superior a la media del país ${ }^{3,5,6}$.

Los casos de leptospirosis asociados a la exposición ocupacional están bien documentados ${ }^{7-10}$, ocurriendo en brotes, así como en trabajadores del área rural, basureros, mineros, limpiadores de desagüe, veterinarios y funcionarios de mataderos, teniendo éstos un mayor riesgo de contaminarse que la población en general. Además, se sabe que brotes en áreas geográficas diferentes pueden presentar variaciones epidemiológicas y clínicas $^{2}$.

En octubre de 1998 fueron diagnosticados los dos primeros casos de leptospirosis en funcionarios de un hospital veterinario, en Rio Grande do Sul, Brasil. Teniendo en consideración su importancia epidemiológica y la falta de reportes semejantes en la literatura médica brasileña, se estudió el comportamiento del primer brote en un hospital veterinario del mencionado estado.

\section{Pacientes y Métodos}

El Hospital Veterinario de la Universidad Federal de Santa María (HV-UFSM), Rio Grande do Sul, Brasil, es un hospital general, regional, que atiende animales de varias especies y de todas las patologías.

En el período de octubre de 1998 a enero de 1999 fueron afectados 37 funcionarios del HVUFSM por un brote de leptospirosis ocupacional. El hospital contaba con 66 funcionarios de varios niveles.

Se realizó un estudio descriptivo de la serie de casos afectados en el brote, siendo analizados los parámetros clínicos y de laboratorio, como así también las variables epidemiológicas.

Los funcionarios fueron atendidos en el servicio de enfermedades infecciosas del Hospital Universitario de Santa María.

Fueron analizadas 37 muestras de suero, tomadas de 37 funcionarios del HV-UFSM durante este período.

El diagnóstico fue sospechado a través de la historia clínica/epidemiológica, asociada a hallaz- gos en exámenes complementarios inespecíficos (hemograma, bilirrubinemia, creatininemia, fosfatasas alcalina, transaminasas hepáticas, protrombinemia, VHS) más serología de leptospira. La confirmación fue efectuada por el Departamento de Microbiología, Sección de Bacteriología de la Universidad Federal de Santa María, laboratorio de referencia en Rio Grande do Sul para serología humana y animal, a través de la técnica de microaglutinación para la pesquisa de aglutininas anti-leptospiras en las muestras (patrón de oro). Además de testar anticuerpos específicos, se empleó en la identificación y clasificación de los serovars aislados.

\section{Resultados}

Estudio serológico. Las muestras serológicas fueron positivas en 32 casos $(86,5 \%)$. Cinco casos $(13,5 \%)$ presentaron cuadro clínico compatible con la enfermedad en asociación con una historia epidemiológica positiva y, a pesar de resultar negativa la serología para leptospira, fueron considerados como casos debido a la situación de brote.

Los seis serotipos encontrados en este brote fueron: bratislava en 25 casos $(43,1 \%)$, australis $24(41,4 \%)$, patoc $5(8,6 \%)$, canicola $2(3,4 \%)$, icterohaemorrhagiae $1(1,7 \%)$ y pyogena $1(1,7 \%)$. La frecuencia de los serovars bratislava y australis fue significativamente superior a los otros.

Variables epidemiológicas. En la Tabla 1 se aprecia la actividad laboral desempeñada por cada uno de los pacientes. La tasa de ataque global de leptospirosis sintomática en la institución fue de $56,6 \%$ (37/66), siendo máxima, $100 \%$, en los funcionarios encargados de la limpieza (9/9) y técnicos del bioterio (3/3), seguido de los guardias con $75 \%$ (3/4) y por los funcionarios de servicios generales con $70 \%$ (7/10).

La edad media fue de 30 años (17-58 años). Veinte de ellos $(54,1 \%)$ eran del sexo masculino.

Manifestaciones clínicas. De los 37 pacientes con diagnóstico de leptospitosis, 22 (59,5\%) presentaron un cuadro sintomático leve. Se constataron los siguientes signos y síntomas en orden de frecuencia: cefalea $(67,5 \%)$; mialgia $(62,1 \%$, $48,6 \%$ generalizada y $37,8 \%$ localizada en las pantorrillas); somnolencia $(54,1 \%)$; mareos $(45,9 \%)$; faringitis $(43,2 \%)$; fiebre $(40,5 \%)$; tos $(37,8 \%)$; linfoadenomegalia $(32,4 \%)$; hiperemia conjuntival $(24,4 \%)$; debilidad $(24,3 \%)$; dolor abdominal (21,6\%); ictericia $(21,6 \%)$; vómitos $(21,3 \%)$; inapetencia $(13,5 \%)$; hemorragia nasal $(10,8 \%)$; poliuria $(10,8 \%)$; exantema $(8,1 \%)$; oliguria $(5,0 \%)$; enterorragia $(2,7 \%)$; hematuria 
$(2,7 \%)$ melena $(2,7 \%)$; hemorragias subconjuntivales $(2,7 \%)$ y petequias $(2,7 \%)$. Ninguno de los pacientes infectados presentó hepatomegalia, esplenomegalia, hematomas ni signos meníngeos (Figura 1).

Los exámenes generales dieron resultados inespecíficos. Ellos fueron importantes para descartar una forma de presentación grave y realizar el diagnostico diferencial. Estos resultados son concordantes con las manifestaciones clínicas poco expresivas (por ejemplo, ictericia en apenas $21,6 \%$ de los casos).
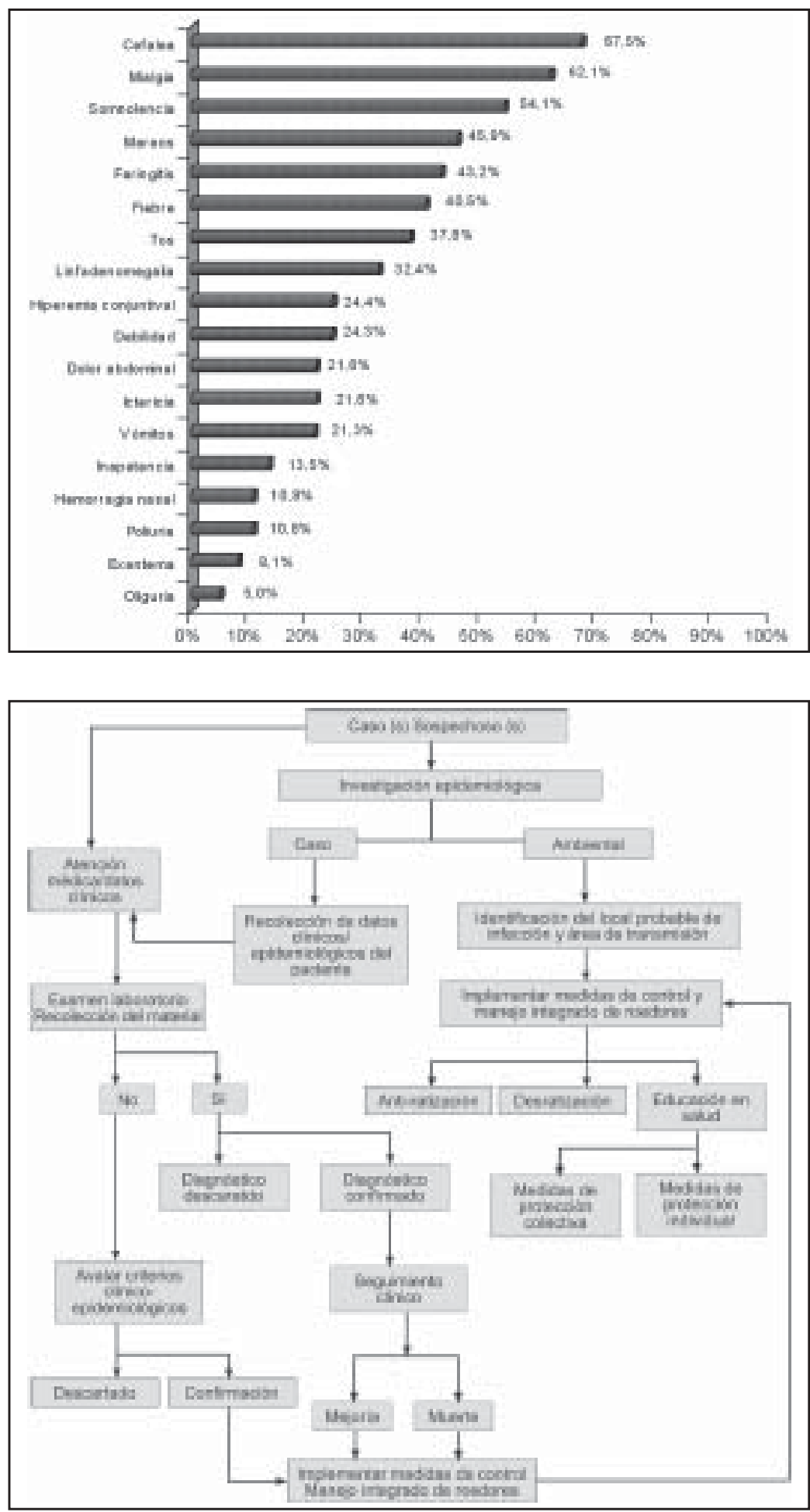

Figura 1. Signos y síntomas encontrados en 37 casos de leptospirosis asociados a la exposición ocupacional atendidos en el HV-UFSM, RS - Brasil.
Figura 2. Algoritmo para la investigación epidemiológica de la leptospirosis recomendado por el Ministerio de Salud de Brasil y la Fundación Nacional de Salud. Fuente: FUNASA, $2003^{14}$. 
Tabla 1. Actividad laboral de 37 pacientes estudiados durante el brote de leptospirosis en el Hospital Veterinario de la Universidad Federal de Santa María - RS - Brasil

\begin{tabular}{lcc}
\hline Ocupación & $\mathbf{N}^{\mathbf{0}}$ & $\boldsymbol{\%}$ \\
\hline Limpieza & 9 & 24,3 \\
Servicios generales & 7 & 18,9 \\
Alumnos de medicina veterinaria & 4 & 10,8 \\
Equipo de enfermería & 4 & 10,8 \\
Técnico de bioterio & 3 & 8,1 \\
Guardia & 3 & 8,1 \\
Recepcionista & 3 & 8,1 \\
Médico veterinario & 3 & 8,1 \\
Chofer & 1 & 2,7 \\
Total & 37 & 100 \\
\hline
\end{tabular}

\section{Discusión}

La Figura 2 muestra el algoritmo de investigación epidemiológica de la leptospirosis utilizado, según recomendaciones del Ministerio de Salud de Brasil y de la Fundación Nacional de Salud brasileña ${ }^{3}$.

El haberse producido un brote en un área limitada, en la cual coexisten varias especies de animales susceptibles a la infección por leptospira, explica la diversidad de serovars encontrados, demostrando la gran prevalencia de animales portadores y diseminadores de la enfermedad en la región ${ }^{5,10-13}$.

En relación al cuadro clínico, se constató un predominio de formas sintomáticas leves, sin ningún caso grave, a pesar de que un paciente presentó el serovar icterohaemorrhagiae, asociado habitualmente a la forma grave de enferme$\operatorname{dad}^{1,4,14,15}$. Podrían haber contribuido en este sentido, una sospecha diagnóstica precoz de la enfermedad y la terapia de soporte adecuada. Es importante comentar la baja prevalencia de pacientes con fiebre en el momento de la consulta, a pesar de que sí había historia de fiebre en los últimos 5 días. El tratamiento consistió en terapia de soporte ${ }^{16}$.

A pesar del conocimiento de esta zoonosis y del riesgo de exposición a ella en una institución como es un hospital veterinario, no existía algoritmo de procedimientos ni acciones relativas al control ambiental de las posibles formas de exposición a esta zoonosis para la población en cuestión. El análisis de este brote permitió ver que su ocurrencia estuvo relacionada a los malos hábitos de higiene tanto del personal como animal. $\mathrm{La}$ mayor prevalencia de leptospirosis entre los trabajadores del aseo del HV-UFSM nos lleva a sospechar que la falta de información sobre las formas de contaminación de la enfermedad sea uno de los factores determinantes ${ }^{7}$. Se confirma así la importancia de la educación continua en servicios para la prevención de enfermedades.

Con la intención de controlar y evitar nuevos brotes se adoptaron medidas y estrategias como procedimientos y normas para el control de leptospirosis en el ambiente hospitalario veterinario, a través de un programa de control de zoonosis intra-hospitalaria.

\section{Leptospirosis: Breve revisión conceptual}

Etiología. Esta enfermedad es causada por microorganismos que pertenecen a la familia Spirochaetaceae y género Leptospira ${ }^{2,6-9,11,12,17-19}$. Las leptospiras son bacterias flexibles (helicoidales), móviles, y que se colorean levemente con la tinción de Gram. Existen dos especies: $L$. biflexa y L. interrogans. Leptospira interrogans es una especie patógena para el hombre y los animales, siendo identificados varios serogrupos que, por su vez, son divididos en más de 200 serovars.

Epidemiología. Es considerada una de las zoonosis más diseminadas y subdiagnosticadas en el mundo; esto se debe en parte a la presentación clínica inespecífica de la enfermedad, así como a la gran capacidad de sobrevida de $L$. interrogans, tanto en animales infectados como en el medioambiente. Esta bacteria tiene la capacidad de habitar en los túbulos contorneados del asa de Henle en animales enteramente asintomáticos, pudiendo sobrevivir hasta seis meses en el medioambiente después de su excreción urinaria $^{3,5,13,20}$

Patogenia en humanos. Inicialmente la leptospira entra a través de la piel y membranas mucosas (lesionadas o no) pudiendo diseminarse por la corriente sanguínea a todo el organismo. El periodo de incubación varía de 24 horas a 28 días, siendo en promedio de 7 a 14 días. La lesión fundamental que explica el cuadro clínico es una vasculitis que compromete los endoteliocitos capilares, causando disfunción renal y hepática, miocarditis y hemorragia pulmonar. Secundariamente se produce extravasación de líquido intravascular que agrava el compromiso renal, y eventualmente desencadena un shock. Los casos fatales presentan hemorragia diseminada de las superficies mucosas y piel $^{21-24}$. La inmunidad adquirida después de la infección es serotipo específica, pudiendo el mismo individuo ser afectado más de una vez por serotipos diferentes. 
Manifestaciones clínicas. La mayoría de las infecciones cursa en forma asintomática o con manifestaciones clínicas inespecíficas y autolimitadas, en el plazo de 4 a 7 días.

La enfermedad evoluciona con o sin ictericia. Forma anictérica (más común) es autolimitada y se divide en dos fases: la primera es llamada fase septicémica y se caracteriza por la presencia de espiroquetas en la sangre, LCR y otros tejidos. Con un inicio súbito, aparece fiebre alta $\left(38^{\circ}\right.$ a $40^{\circ}$ ) asociada a cefalea con intensidad leve a moderada, calofríos, mialgia (en particular en pantorrillas), tos, dolor torácica, rigidez de cuello e inyección conjuntival. En los casos más graves puede haber hepato-esplenomegalia, pancreatitis, compromiso renal y manifestaciones respiratorias (hemoptisis y tos seca). Ese cuadro dura cerca de 4 a 7 días siendo seguido por un período de mejoría de 2 días, tras el cual se inicia la segunda fase de la enfermedad. En la segunda fase-fase inmune- las leptospiras desaparecen de la sangre mientras son detectables anticuerpos específicos circulantes. No siempre esa fase acontece, mas, cuando está presente, se manifiesta como un cuadro de meningitis aséptica caracterizada por ausencia de leptospiras y presencia de anticuerpos en el LCR. Este cuadro puede durar de 4 a 30 días $3,4,14,25,26$. Forma ictérica. Es la presentación grave de leptospirosis (5 a 10\% de los casos), conocida como enfermedad o síndrome de Weil. Cursa con ictericia, hemorragia e insuficiencia renal aguda y está asociada al serotipo ictehaemorrahagiae ${ }^{1,23,27,28}$. Pueden ocurrir alteraciones hepáticas con discreto aumento de las transaminasas ( raciones renales incluyen aumento de uremia y creatininemia, leucocituria, hematuria, cilindruria y proteinuria. La letalidad de esa forma ictérica es de aproximadamente 5 a 20\%. Todas las manifestaciones pulmonares, hepáticas y renales causadas son reversibles ${ }^{4,21,29,30}$

Diagnóstico. Está basado en la historia, cuadro clínico y en los resultados de los exámenes complementarios como anemia, leucocitosis con desviación a izquierda, trombocitopenia, elevación de creatinfosfoquinasa y leucocituria. Son resultados inespecíficos pero que, asociados al cuadro clínico, sugieren el diagnóstico. La confirmación diagnóstica se logra menos frecuentemente por métodos directos (pesquisa mediante microscopia de campo oscuro o cultivo) y, en la mayoría de los casos, por métodos serológicos (reacción de microaglutinación, tests de FC y EIA). Sin embargo, el test más recomendado y confiable actualmente es la reacción de aglutinación ${ }^{4,6,10-12}$.
Tratamiento. Se basa en los cuidados generales del enfermo y administración de penicilina, principalmente en la fase inicial ${ }^{16}$.

Pronóstico. Generalmente es bueno, pero depende invariablemente, de la susceptibilidad del hospedero y de la forma clínica desarrollada ${ }^{16}$. La letalidad de la enfermedad depende de las siguientes variables: gravedad de la forma clínica, diagnóstico y tratamiento precoz, edad del enfermo, pudiendo llegar hasta $40 \%$ en los casos graves $^{3}$.

Profilaxis.Las medidas de prevención deben orientarse hacia el control de reservorios (ratas y ratones), a través de acciones permanentes de anti ratización y des ratización; medidas de protección individual y colectiva de la población humana y animal (doméstica) en riesgo; y mejoramiento medioambiental e higiénico-sanitario de las poblaciones en áreas endémicas, como el área geográfica en cuestión ${ }^{3,4}$. Las acciones educativas permanentes para el individuo y la colectividad contemplan contenidos sobre el modo de transmisión, signos y síntomas de la enfermedad; orientar trabajadores en situación de riesgo a utilizar equipos de protección individual, tales como máscaras, guantes, botas y en ausencia de éstos, bolsas de polietileno cubriendo las manos y los pies del trabajador; orientar sobre el consumo de agua filtrada, hervida o clorada, manteniendo limpios los estanques de agua; limpiar y desinfectar el medioambiente y vacunar anualmente los animales domésticos (perros y bovinos), en regiones endémicas ${ }^{3}$.

\section{Resumen}

Este estudio describe un brote de leptospirosis humana asociado a la exposición veterinaria en el Hospital Veterinario de la Universidad Federal de Santa María, Río Grande do Sul, Brasil, acaecido entre octubre de 1998 y enero de 1999. Fueron detectado 37 casos siendo confirmados serológicamente 32 de ellos. Los serovars más encontrados fueron la L. bratislava en 25 muestras $(43,1 \%)$ y L. autralis en $24(41,4 \%)$. Individuos de diferentes actividades se contaminaron durante este brote, $24,3 \%$ eran trabajadores del aseo. Se concluyó que los casos de leptospirosis entre los trabajadores del aseo del Hospital Universitario, fueron favorecidos por falta de información sobre el modo de transmisión de esta zoonosis y de capacitación para evitar la exposición ocupacional.

\section{Agradecimientos}

A la enfermera Hna. Mirta García por la traducción del texto al idioma español. 


\section{Referencias}

1.- Costa E, Costa Y A, López A A, Sacramento E, Bina J C. Formas graves de leptospirose: aspectos clínicos, demográficos e ambientais. Rev Soc Bras Med Trop 2001; 34: 261-7.

2.- Dabanch P J. Zoonosis. Rev Chil Infectol 2003; 20: 47-51.

3.- FUNASA. Fundação Nacional de Saúde. Guia de vigilância epidemiológico. Ministério do Brasil: Brasília, 2003.

4.- Vinetz J M. Leptospirosis. Curr Opin Infect Dis 2001; 14: 527-38.

5.- Favero A C M, Pinheiro S R, Vasconcellos S A, Morais Z M, Ferreira F, Neto J S F. Sorovares de Leptospiras predominantes em exames sorológicos de bubalinos, ovinos, caprinos, eqüinos, suínos e cães de diversos estados brasileiros. Ciência Rural 2002; 32: 613-9.

6.- Barcellos C, Lammerhirt C B, Almeida M A B, Santos E. Distribuição espacial da leptospirose no Rio Grande do Sul, Brasil: recuperando a ecologia dos estudos ecológicos. Cad Saúde Pública. 2003; 19: 128392.

7.- Almeida L P, Martins L F S, Brod C S, Germano P M L. Levantamento sorepidemiológico de leptospirose em trabalhadores do serviço de saneamento ambiental em localidade urbana da região sul do Brasil. Rev Saúde Pública 1994; 28: 76-81.

8.- Ciceroni L, Pinto A, Benedetti E, Pizzocaro P, Lupidi R, Gelosa L, et al. Human letospirosis in Italy:19861993. Eur J Epidemiol 1995; 11: 707-10.

9.- Rao S R, Gupta N B, Agarwal S K. Leptospirosis in India and the rest of the world. Braz J Infect Dis 2003; 7: 178-93.

10.- Romero E C, Bernardo C C M, Yasuda P H. Human leptospirosis: a twenty-nine-year serological study in São Paulo, Brazil. Rev Inst Med Trop S Paulo 2003; 45: 245-8.

11.- Figueiredo C M, Mourão A C, Oliveira M A A, Açves W R, Ooteman M C, Chamone C B, Koury M C. Leptospirose humana no município de Belo Horizonte, Minas Gerais, Brasil: uma abordagem geográfica. Rev Soc Bras Med Trop 2001; 34: 331-8.

12.- Garcia J L, Navarro I T. Avaliação sorológica da leptospirose e brucelose em pacientes moradores da área rural do município de Guaraci, Paraná, Brasil. Rev Soc Bras Med Trop 2001; 34: 299-300.

13.- Schimidt V, Arosi A, Santos A R. Levantamento sorológico da leptospirose em caprinos leiteiros no Rio Grande do Sul, Brasil. Ciência Rural 2002; 32: 609-12.

14.- Silva H R, Tavares-Neto J, Bina J C, Meyer R. Leptospirose-infecção e forma subclínica em crianças de Salvador, Bahia. Rev Soc Bras Med Trop 2003; 36: 227-33.

15.- Travejo R T, Rigau-Perz J G, Ashford D A et al. Epidemic leptospirosis associated with pulmonary hemorrage - Nicaragua, 1995. J Infect Dis 1998; 178: 1457-63.

16.- Costa E, Lopes A A, Sacramento E, Costa Y A, Matos E D, Lopes M B, Bina J C. Penicillin at the late stage of leptospirosis: a randomized controlled trial. Rev Inst Med Trop S Paulo 2003; 45: 141-5.

17.- Chu K M, Rathinan R, Namperumalsamy P, Dean D. Identification of leptospira species in pathogenesis of uveitis and determination of characteristics in South India. J Infect Dis 1998; 177: 1314-21.

18.- Kuriakose M, Eapen C K, Paul R. Leptospirosis in Kolenchery, Kerala, India: epidemiology, prevalent local serogroups and serovars and a new serovar. Eur $\mathbf{J}$ Epidemiol 1997; 13: 691-7.

19.- Perrocheau A, Perolat P. Epidemiology of leptospirosis in New Caledonia (South Pacific): a one-year survey. Eur J Epidemiol 1997; 13: 161-7.

20.- Secretaria da Saúde e do Meio Ambiente - Brasil. Ações em Saúde: Zoonoses e Vetores. Normas técnicas e operacionais. Secretaria de Saúde de Meio Ambiente. 1997.

21.- Clase G, Juana F, Paulino B, Rosa M, et al. Insuficiência renal aguda em pacientes internados com diagnóstico de leptospirose. Rev Bras Med 1996; 53: 347-62.

22.- Martins M G, Matos K T, Silva M V, Abreu M T. Ocular manifestations in the acute phase of leptospirosis. Ocul Immunol Inflam 1998; 6: 75-9.

23.- Salto Glu, Naksu Hz, Tasova Y, Arslan A, et al. Leptospirosis: twelve Turkish patients with the Weil' syndrome. Acta Med Okayama 1997; 51: 339-42.

24.- Torres R, Jaime R, Isturiz R, Mondolfi A G et al. Historia natural de la leptospirosis humana en Venezuela. Bol Venez Infectol 1989; 1: 27-9.

25.- Abdulkader R C R M, Daher E F, Camargo E D, Spinosa C, Silva M V. Leptospirosis severity may be associated with the intensity of humoral immune response. Rev Inst Med Trop S Paulo 2002; 44: 79-83.

26.- Bezerra H M, Ataíde Junior L, Hinrichsen S, Travassos F M, Travassos P T C, Silva M B. Comprometimento do sistema nervoso na leptospirose: Avaliação dos aspectos neurológicos. Arq Neuropsiquiatria 1993; 51: 1457-63.

27.- Daher E D F, Brunetta D M, Silva Junior G B S, Puster R A, Patrocínio R M S V. Pancreatic involvement in fatal human leptospirosis: clinical and histopathological features. Rev Inst Med Trop S Paulo 2003; 45: 307-13

28.- Hill M K, Sanders C V. Leptospiral pneumonia. Sem Resp Infect 1997; 12: 44-9.

29.- Perani V, Farina C, Maggi L, Moioli F, Pizzocaro P, Pugliese C. Pneumonia due to Leptospira ssp.: results of an epidemiological and clinical study. Int J Tuberc Lung Dis 1998; 2: 766-70.

30.- Silva J J P, Dalston M O, Carvalho J E M, Setúbal S, Oliveira J M C, Pereira M M. Aspectos clínicos-patológicos e imuno-histoquímicos da forma pulmonar grave da leptospirose. Rev Soc Bras Med Trop 2002; 35 : 391-5.

Correspondencia a:

Marcelo Carneiro

carneiromarcelo@yahoo.com.br 